\title{
El proceso de partenariado en el proyecto de aprendizaje servicio del Banco de Sangre y Tejidos
}

\author{
Mariona Graell Martín mgraell@uic.es \\ Universidad Internacional de Catalunya, España.
}

\section{Resumen}

El aprendizaje servicio es una herramienta que vincula las entidades tanto educativas como sociales con un doble objetivo: formar a los futuros ciudadanos de forma democrática, cívica, participativa y consciente de la realidad, y dar respuesta a unas necesidades sociales. Para que esto sea posible se hace indispensable tratar uno de los aspectos más importantes de este tipo de prácticas, las relaciones de partenariado. Este documento, mediante la metodología de la investigación acción, trata de dar unas pautas a las organizaciones sociales para que estas puedan llegar a las instituciones educativas y crear proyectos de aprendizaje servicio. El diseño y la implantación del proyecto "Donación de sangre y educación para la ciudadanía" realizado en y desde el Banco de Sangre y Tejidos durante cinco años nos permiten analizar y desarrollar este objetivo. El resultado de dicha propuesta es la elaboración de un protocolo de actuación que orienta a las instituciones sociales para elaborar proyectos de aprendizaje servicio conjuntamente con las entidades educativas. Estas primeras aproximaciones a la temática facilitarán a las entidades sociales que por primera vez quieran vincularse de forma ordenada con las entidades educativas para el desarrollo de un proyecto de aprendizaje servicio.

\section{Palabras clave}

Interacción social, comunidad, educación cívica, método de proyectos, aprendizaje servicio. 


\title{
El proceso de partenariado en el proyecto de aprendizaje servicio del Banco de Sangre y Tejidos
}

\begin{abstract}
Service-learning is a tool that links educational institutions and social organisations with a dual objective: to train future citizens in a democratic, civil, participatory and conscious way; and to respond to social needs. To make this possible it is essential to address one of the most important aspects of these practices, partnership. This paper, using the methodology of action research, provides guidance to social organizations so that they can create educational and service-learning projects. The design and implementation of the project, Blood Donation and civic education, conducted over the course of five years, from the Blood and Tissue Bank will allow us to analyse and develop this objective. The result of this proposal is to develop a protocol that guides social institutions who want to develop service learning projects in conjunction with educational institutions. This approach, for the first time, will help facilitate a connection between social institutions interested and educational institutions with the goal of developing a service-learning project.
\end{abstract}

\section{Keywords}

Social Interaction, Community, Civic Education, Project Based Learning, ServiceLearning. 


\section{Introducción}

Formar a personas para que sean capaces de poder relacionarse con los demás y se sientan pertinentes a la comunidad en la que viven es uno de los principales objetivos de la educación. Si a este propósito le sumamos que además han de poderse conocer a sí mismos y discernir lo que quieren o no hacer, podríamos hablar de educación en valores. Se desea formar a unos ciudadanos democráticos, críticos, participativos y conscientes de su realidad. Y se puede hacer de dos maneras: hablando hasta hacer alumnos especialistas en oratoria (Larrauri, 2012) o actuando en la realidad y reflexionándola. El aprendizaje servicio podría ser un ejemplo de esta segunda perspectiva educativa. Se aprende a la vez que se da un servicio a la comunidad. Este servicio no repercute exclusivamente en las personas que la realizan, sino que en múltiples ocasiones se sitúa en un contexto social en que las instituciones y organizaciones implicadas necesitan ponerse de acuerdo entre ellas para realizar un proyecto en común. De este modo, el aprendizaje servicio requiere en distintas situaciones cuestiones vinculadas con el partenariado -concepto que define las relaciones de colaboración que se crean entre dos o más personas u organizaciones-. Así, en los proyectos de ApS las colaboraciones que se establecen pueden ser complejas e incontrolables. Es por esto que a partir de las múltiples aplicaciones del proyecto creado para el Banco de Sangre y Tejidos de Cataluña se presenta el proceso de partenariado que le ha sido necesario y le sigue funcionando a esta institución social. Las redes sociales, las conexiones y las alianzas que aparecen mediante los proyectos de aprendizaje servicio mejoran el entorno en el que se vive y de las personas que se implican (Tapia, 2006). Mediante el proyecto donación de sangre y educación para la ciudadanía se mejora la cantidad de donaciones así como la conciencia social de la necesidad de la donación de sangre.

\section{El aprendizaje servicio (ApS)}

Hasta finales del S. XX e inicios del S. XXI las instituciones se han ido relacionando de forma horizontal y vertical, siguiendo esquemas estructurados en la que su distribución ha sido inamovible e intocable. Esto ha repercutido en que las relaciones intra e interorganizacionales siguieran un orden jerárquico concreto y preestablecido. Con el tiempo, los organigramas jerárquicos van desapareciendo para dar lugar a nuevas estructuras que interconectan campos dispares y que se implican en nuevas áreas de acción. Una de ellas, desde la perspectiva educativa, son las experiencias de aprendizaje servicio.

Es una actividad pedagógica que tiene gran contenido educativo y social. Es por ello que se considera fundamental reflejar cuáles son sus principales características. Seguidamente se busca describir los siete rasgos que son definitorios del aprendizaje 


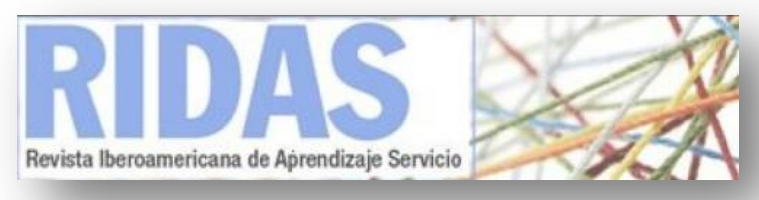

servicio (Puig, Batlle, Bosch y Palos, 2006): 1) Un proyecto educativo con utilidad social. Da respuesta a unas problemáticas emergentes y que, por lo tanto, hacen que las acciones que se realizan sean útiles e innovadoras para la comunidad. El diseño de ellas tiene una estructura muy concreta: definición del reto, generación de la ideas, diseño de un plan, llevarlo a cabo y evaluación de los resultados. 2) Un método para una educación formal y no formal, para todas las edades y que debe tener un espacio temporal concreto. El aprendizaje servicio es una propuesta lo suficientemente flexible como para que se acomoden tanto la educación formal como la no formal. Los implicados han de poder responsabilizarse en todo el proceso que siguen e ir tomando conciencia de los conocimientos que se van asumiendo. 3) Un servicio para aprender y colaborar en un marco de reciprocidad. El servicio pone en evidencia la relación de dos aspectos importantes en el aprendizaje servicio -el conocimiento y la ciudadanía-. Los conocimientos que se han adquirido a partir de la conciencia de unas necesidades reales de la comunidad, es lo que motiva a realizar las acciones como miembros de la sociedad. 4) Un proceso de adquisición de conocimientos y competencias para la vida. El aprendizaje servicio posibilita a todas las personas que llevan a cabo una experiencia de éstas a trabajar un alto grado de conocimientos significativos, adquiriendo competencias, habilidades y valores que permiten el desarrollo humano. 5) Un método de pedagogía activa y reflexiva. El aprendizaje servicio se lleva a cabo mediante una metodología de aprendizaje experiencial (Bertrand y Valois, 1995, 132) Así pues, es condición sine qua non que las personas implicadas participen activamente en el proceso y que tomen la acción como propia mediante la reflexión. 6) Una red de partenariado y de instancias de colaboración y apoyo. Los proyectos de aprendizaje servicio requieren de alianzas entre diferentes personas $u$ organizaciones que compartan un mismo objetivo. La complejidad del aprendizaje servicio radica justamente ahí, puesto que en diferentes ocasiones las realidades que se implican no siempre son del mismo ámbito y esto puede dificultar la labor. 7) Un impacto formativo y transformador múltiple. Un impacto formativo porque las personas que intervienen aprenden múltiples aspectos que sin el proyecto no lo hubieran logrado. $Y$ un impacto transformador porque después de actuar en la realidad y de pretender atenuar la necesidad detectada, hay algo que se ha modificado mejorando la sociedad.

\section{Propósito: del ámbito social al educativo}

Los proyectos de aprendizaje servicio requieren de vínculos para poder realizarlos. El servicio, en la medida de lo posible, se ha de dirigir a un colectivo de personas ajenas al grupo que lo realiza. Esta condición hace imprescindibles las alianzas entre organizaciones de diferentes ámbitos. Las relaciones de partenariado, como se ha señalado anteriormente, requieren de múltiples factores como el conocimiento y reconocimiento, la confianza mutua, la responsabilidad compartida, el respeto de los tiempos de cada institución, los lenguajes de cada ámbito, entre otros. Este conjunto

Graell, M. (2015). El proceso de partenariado en el proyecto de aprendizaje servicio del Banco de Sangre y Tejidos. RIDAS, Revista Iberoamericana de Aprendizaje y Servicio, 1, 76-90. DOI 10.1344/RIDAS2015.1.5 
de elementos se hace preciso respetarlos y ordenarlos, pero ¿cómo hacerlo? Tradicionalmente hemos visto como las instituciones educativas se han vinculado con algunas organizaciones sociales. Pero esta forma de trabajar -del ámbito educativo al social-, aunque sea la pauta más habitual, no es la única. Podemos imaginar una variante: que las organizaciones sociales a partir de una detección de necesidades sociales acudan a los centros y entidades educativas para solicitar su ayuda y participación -del ámbito social al educativo-.

Una de las preocupaciones que quisiéramos abordar en este artículo es cómo las organizaciones no educativas puedan llegar a las instituciones educativas sin que éstas se sientan invadidas. Los proyectos de aprendizaje servicio precisan de unas relaciones que requieren de una serie de pasos para que la propuesta sea viable. Las instituciones vinculadas han de poder salir ganando de forma independiente. Es por ello que la pregunta a la que daremos respuesta a lo largo de este artículo es ¿cómo nos dirigimos desde las entidades no educativas a las educativas mediante los proyectos de aprendizaje servicio? Creemos que esta cuestión es clave y que se presenta cada vez que una organización, entidad o institución no educativa quiere trabajar con y mediante el mundo educativo a partir del aprendizaje servicio.

\section{Banco de Sangre y Tejidos}

Para responder al interrogante que nos formulamos anteriormente, hemos trabajado con una organización que ha cumplido este propósito y nos ha permitido elaborar la sistematización de este proceso. El Banco de Sangre y Tejidos es una empresa pública que tiene el objetivo de gestionar y administrar la donación, la transfusión y el análisis de la sangre y el plasma sanguíneo. Por sí misma, es una empresa que funciona como cualquier otra -ingresos y gastos-, pero su mayor peculiaridad es que la materia prima la obtiene del voluntariado, es decir, de la donación gratuita y voluntaria de personas anónimas. Este factor hace que se vincule la donación a un hecho solidario, voluntario y gratuito.

Con el continuo crecimiento de la población y la falta de conciencia social de que la sangre es una necesidad, el Banco de Sangre se encuentra ante la dificultad de abastecer a todos los centros sanitarios de los productos sanguíneos que requieren. Se encarga un estudio en el departamento de marketing para saber el motivo por el cual la comunidad no participa. Los resultados de dicho estudio muestran el desconocimiento del Banco de Sangre y la falta de conciencia social sobre la necesidad de la donación de sangre. Se concluye que es necesario dar a conocer esta realidad y pedir una mayor participación. Así pues, lo que se busca es presentar la donación de sangre como un acto cívico y cotidiano. 
Ante esta realidad, el equipo del Banco de Sangre valora las posibilidades de actuación, y entre ellas aparece el aprendizaje servicio. Este se vinculará al departamento de marketing en primer lugar, para el diseño de un posible proyecto y posteriormente en la unidad de promoción. Será un proyecto piloto durante los primeros años y si tiene buenos resultados, se incidirá en él mediante mayor implicación por el propio interés de la organización: recaudar mayor número de bolsas de sangre. Uno de los elementos que le permite al Banco de Sangre trabajar en un proyecto vinculado con la educación es que de él proceden conceptos y técnica que son ricos en contenido y pueden ofrecer y enriquecer a las entidades educativas. Por el contrario, las instituciones educativas pueden ayudar a concienciar a la comunidad próxima de la necesidad de la donación de sangre, además de recaudarla.

A partir de este conjunto de posibilidades aparece el proyecto Donación de sangre y educación para la ciudadanía. Se dirige a menores de edad, que no pueden dar sangre, pero ayudan a difundir la importancia de la donación y, en caso que quieran, proporcionarles la posibilidad de que en un futuro puedan ser donantes. Así pues, se les forma en el ámbito medico científico, de comunicación y de valores, para que creen una campaña de sensibilización y promoción de la donación de sangre. Una vez realizadas este conjunto de acciones, el Banco de Sangre queda un día con la entidad educativa, para posibilitar que las personas sensibilizadas puedan dar sangre. Una actividad que podría quedarse en sensibilizar a los jóvenes de la necesidad de productos sanguíneos, se transforma en un proyecto de aprendizaje servicio en que los participantes se encargan de hacer una campaña de comunicación desarrollando su creatividad, ayudando a otras personas que lo necesitan y participando activamente en la sociedad que les rodea. Aparece la oportunidad de ser responsables socialmente y esto les hace más consciente de la comunidad en la que viven.

\section{Aspectos metodológicos del proyecto}

En toda investigación se plantean una serie de retos que se van resolviendo a lo largo de su proceso. Lo que se busca en ésta es inspirar a las organizaciones sociales sobre cómo llegar a las instituciones educativas para realizar proyectos de aprendizaje servicio. En este sentido, se busca determinar los procesos de partenariado necesarios para vincular el área social con la educativa. Por lo tanto, lo que se trata es concretar y analizar los procesos a través de los cuales una organización no explícitamente educativa aplica un programa de aprendizaje servicio con las entidades educativas. $Y$ para poder hacerlo se ha realizado a partir de la metodología de la investigación acción que posibilita el diseño, la implantación y la evaluación. Éste método ha permitido observar, analizar y sistematizar el proceso por el cual un proyecto de aprendizaje servicio se lleva a cabo. Este marco metodológico nos deja participar activamente en el desarrollo de la investigación a la vez que se estudia y se reflexiona. Tal como afirma 
Kemmis y McTaggart (1988) la investigación acción es una forma de indagación autoreflexiva realizada por quienes participan en las situaciones sociales y educativas para la mejora de sus propias prácticas, a la vez que las comprenden. Este proceso de investigación ha constado de cuatro etapas: la primera, conocimiento y análisis del contexto; la segunda, diseño del programa; la tercera, las pruebas piloto del proyecto; y la cuarta y última, el impulso e implantación del proyecto en el Banco de Sangre y Tejidos. Para ello se ha seguido un patrón idéntico en cada etapa que ha permitido ir avanzando en el proceso y substraer los resultados. En el cuadro que se muestra a continuación se puede observar como la estructura se repite de forma lineal y a su vez, en una espiral que permite avanzar en el proceso de la investigación. Cada etapa se inicia con una finalidad y unos objetivos que delimitarán el rumbo del proceso de investigación mediante una serie de interrogantes que se van resolviendo en la medida en que el proceso avanza. Después de diseñar en qué consistirá la etapa, se pasa la fase de la aplicación, en la que entra la planificación y el propio desarrollo. En este periodo, es importante usar unas buenas técnicas de recogida de datos para que, posteriormente, se pueda evaluar, mediante la sintetización y la reflexión sobre todos los procesos que han ido ocurriendo. Es por ello que en la última fase de cada etapa se valora todo aquello que ha sucedido en el proceso y se obtienen unas conclusiones para la siguiente etapa. Por lo tanto, todo un proceso de investigación acción que ha tenido una duración aproximada de cinco años.

Del diseño y aplicación de un proyecto llevado a cabo en 132 ocasiones, 81 en el ámbito formal y 51 en el no formal (Graell, 2010), se extrae el resultado que se presenta a continuación. Ordenar los diversos elementos que han ido surgiendo a lo largo de la implementación del proyecto nos ha permitido determinar un protocolo que pueda servir de inspiración a situaciones similares a la que se presenta.

\section{Resultados: protocolo de actuación}

Mediante la aplicación experimental del proyecto -Donación de sangre y educación para la ciudadanía - se va confeccionado el protocolo de actuación y fijando el modo de desarrollo en cada situación concreta. Este permite determinar cuáles son los pasos que son necesarios para el desarrollo del programa en una institución educativa. Se presenta como resultado puesto que es uno de los aspectos más importantes en la aplicación de un proyecto de aprendizaje servicio. Los pasos del programa han sido sistematizados con la intención deliberada de favorecer posibles prácticas similares de otras entidades sociales, parecidas al Banco de Sangre.

Ante la voluntad de pensar en el desarrollo de un protocolo, un aspecto que deberíamos cuestionarnos previamente es ¿qué entendemos por protocolo? En su definición más primitiva (R.A.E) se denomina protoculum a la primera hoja de un 


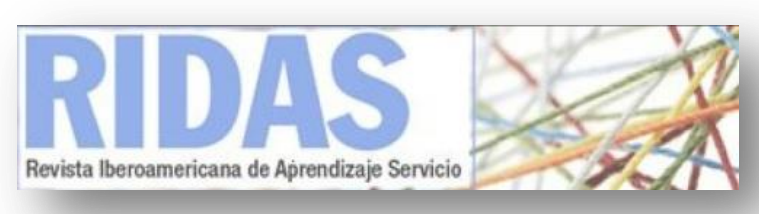

escrito y en ésta primera se encuentran las directrices sobre las que se rige un documento. Esta inicial aproximación determina las que se han hecho a posteriori: de las cinco acepciones que se encuentran podríamos recoger la que creemos que se adecúa más a lo que proponemos: "Serie ordenada de escrituras matrices y otros documentos que un notario o escribano autoriza y custodia con ciertas formalidades". Así pues, si lo adaptamos al ámbito educativo podemos decir que es aquel conjunto de elementos que ordenados y escritos pautan los pasos a seguir en una acción. De esta forma, lo que se pretende en este documento es redactar las pistas necesarias para que las personas que quieran llevar a cabo por primera vez un proyecto de aprendizaje servicio puedan hacerlo con menor dificultad y mayor exactitud.

Entendemos que las propuestas de aprendizaje servicio tienen una estructura parecida entre ellas. Es por ello que pensamos que un protocolo de actuación puede orientar a aquellas acciones que podrían parecer complejas llevadas a la práctica. Ante los múltiples factores que intervienen en éstos proyectos, el protocolo que se presenta intenta sugerir el desarrollo de una actividad de la mejor manera posible mediante pasos muy precisos. El documento que planteamos tiene cuatro etapas que aparentemente pueden ser las clásicas de todo proyecto, pero que en su interior radica la diferencia y la concreción del aprendizaje servicio. Inicialmente se establece el primer contacto con el centro. La segunda se caracteriza por la concreción del proyecto en el centro. La tercera etapa explica el desarrollo del proyecto en los grupos de jóvenes. Y la cuarta y última se evalúa la puesta en marcha del proyecto y se prevé su continuidad.

\begin{tabular}{|l|}
\hline Etapas de actuación \\
\hline Establecer el primer contacto con el centro \\
Concreción del proyecto en el centro \\
Desarrollar el proyecto con los grupos de infantes y jóvenes \\
Evaluar el desarrollo del proyecto y prever su continuidad \\
\hline
\end{tabular}

Tabla 1. Etapas de actuación. Fuente: elaboración propia.

\section{Establecer el primer contacto con el centro}

En este primer periodo la entidad social que impulsa el proyecto tiene como objetivo llegar a las entidades educativas, mostrar el programa y plantear la posibilidad de desarrollarlo. Para ello hay una serie de aspectos necesarios para lograr éste propósito que se deben de tener en cuenta. A continuación se explican de forma ordenada según la secuencia que se seguiría. 


\section{RDAS}

Establecer el primer contacto con el centro

Informar a la dirección del centro sobre el proyecto

Decidir y acordar la colaboración.

Fijar una persona de referencia del centro

Acordar un trabajo conjunto y, si es posible introducirlo en el PEC

Solicitar el consentimiento de los padres

Tabla 2.Establecer el primer contacto. Fuente: elaboración propia.

Informar a la dirección del centro sobre el proyecto. En la primera fase se hace indispensable comprender que el centro educativo no tiene por qué conocer a la entidad social que quiere desarrollar el proyecto. Es por este motivo que se considera de crucial importancia presentar la entidad social y la existencia de una actividad educativa que puede interesar a la dirección del centro.

Decidir y acordar la colaboración. El segundo aspecto a tener presente es como se decide y se acuerda la colaboración, en el caso que se realice. Para garantizar el buen funcionamiento del proyecto, es importante que todas las personas que quieran implicarse lo hagan de forma autónoma y voluntaria.

Fijar una persona de referencia del centro. La tercera fase trata de fijar una persona de referencia del centro educativo. Esta persona será la que permanecerá y desarrollará, conjuntamente con el referente de la entidad social, el proyecto. En este momento se crea el equipo de trabajo entre los referentes de ambas entidades. Se determinan las formas de comunicación más factibles y que permitan mayor fluidez. Cada centro y persona tiene sus peculiaridades y no siempre el teléfono es el medio más adecuado.

Acordar un trabajo conjunto y, si es posible introducirlo en el PEC. La cuarta fase trata de acordar la colaboración y buscar cómo introducir el proyecto en el centro. Es recomendable que se introduzca en el proyecto educativo del centro o en el plan de actuación de éste para garantizar que el programa esté conectado y no sea una actividad aislada.

Solicitar el consentimiento de los padres. El quinto momento se preocupa por proporcionar un documento de consentimiento de los padres, y otro sobre la protección de los derechos de imagen de los participantes. Los proyectos de aprendizaje servicio en algunas ocasiones tratan temas que pueden ser controvertidos para los padres, y sin su autorización puede influir negativamente en su aplicación. Los derechos de imagen es otro aspecto relevante, pues muchos de las actuaciones que se 


\section{RDAS}

realizan se fotografían para tener documentación gráfica de la movilización y transformación del entorno y sus participantes.

\section{Concreción del proyecto en el centro}

Una vez que el centro ya ha aceptado desarrollar el proyecto, se hace indispensable adaptarlo a sus necesidades. Es por ello, que los proyectos de aprendizaje servicio que se quieran replicar en múltiples centros educativos han de poder ser flexibles, para luego poderse acomodar en la realidad y sus circunstancias. Para poderlo hacer es imprescindible una serie de pasos.

\begin{tabular}{|l|}
\hline Concreción del proyecto en el centro \\
\hline Concretar el proyecto en base a los requisitos del centro \\
Establecer un documento guía que permita el seguimiento de todos los acuerdos que se establezcan \\
\hline
\end{tabular}

Tabla 3. Concreción del proyecto en el centro. Fuente: elaboración propia.

Concretar el proyecto en base a los requisitos del centro. A qué destinatarios se dirigirá, que servicio son capaces de asumir, qué tipo de formación se hace necesaria realizar o cuál es el calendario más oportuno. Según cada uno de estos aspectos, el programa que se llevará a cabo tomará una forma u otra.

Informar de la realización del proyecto a los padres y al claustro. Una vez que el proyecto se adecúa a las perspectivas y necesidades, tanto de la entidad educativa como de la social, se presenta al equipo de profesionales de la educación. De esta forma todo el centro sabrá de la existencia del proyecto y proporcionará su ayuda si lo cree conveniente. Es importante que todo el equipo de educadores o docentes sepan de la presencia de este proyecto, para que no sea una actividad aislada y en caso que lo crean oportuno puedan integrarlo en sus propuestas educativas.

Establecer un documento guía que permita el seguimiento de todos los acuerdos que se establezcan. En este periodo se debe hacer un documento guía que refleje todos los aspectos que se han ido planificando hasta el momento. De esta manera, quedarán por escrito los acuerdos que se han programado. Permitirá visualizar la formación que se haya pactado, con unos contenidos específicos según las necesidades educativas de los participantes. Este primer momento tendrá lugar unos días y unas horas en las que quedará pautado en el documento. También quedará ilustrada la preparación de las acciones de servicio y la temporalización que tendrán. Otro elemento que se muestra en esta planificación es cómo está programado el servicio, es decir, que tipo de 


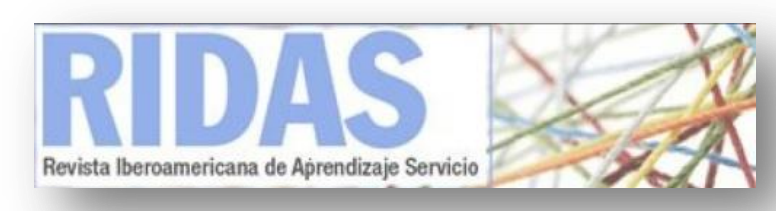

acciones se consideran las más apropiadas. Asimismo se recomienda que a lo largo del proceso se disponga de espacios de reflexión de forma transversal y final. En este último es donde se expresan las opiniones de los participantes y a la vez se les agradece su participación.

\section{Desarrollar el proyecto con los grupos de niños y jóvenes}

Cuando el periodo de planificación ha llegado a su fin, aparece uno nuevo que trata de llevar a cabo todos los aspectos que se han programado. Para ello se hace indispensable una serie de pasos que a continuación se exponen.

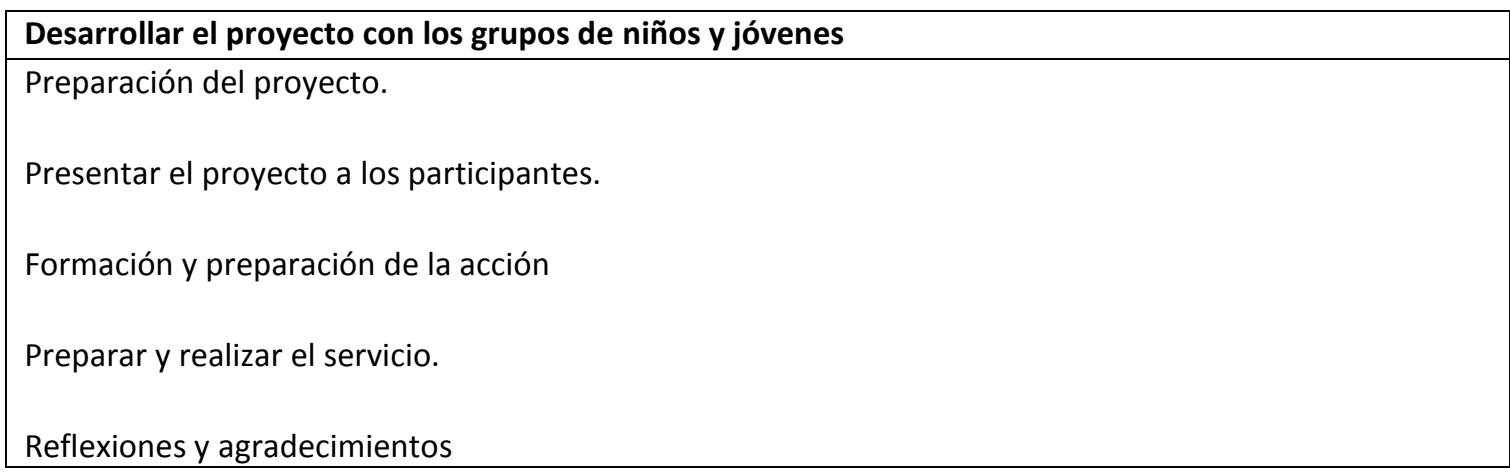

Tabla 4. Desarrollar el proyecto con los grupos de niños y jóvenes. Fuente: elaboración propia.

Preparación del proyecto. En este instante aparece en primer lugar la preparación de la formación, teniendo en cuenta todos los aspectos que hasta el momento se han pactado con el centro educativo. Tenemos que pensar en si es un centro de educación formal o no formal, si la formación ha de ser más o menos dinámica, a qué tipo de destinatarios va dirigida la formación, qué tipo de contenidos son capaces de adquirir, o qué materiales son los más adecuados para que asuman mejor los conocimientos necesarios para una acción.

Presentar el proyecto a los participantes. En segundo lugar aparece el momento de presentar el proyecto a las personas a quienes se dirige el programa. Este momento es clave, puesto que dependerá cómo el proyecto aparezca en las vidas de los participantes, de que éstos quieran responsabilizarse o no. Esta situación influirá en el sentimiento de pertinencia que puedan tener del proyecto, es decir, que se lo sientan suyo y propio. Todo ello dependerá del tipo de participación que se promueva (Trilla y Novella, 2001, 143). En este instante es importante recordar que mediante una mayor implicación de los participantes en el proyecto se lo sentirán propio, propiciando así el sentimiento de pertinencia respecto al proyecto y a las acciones que se realizan; y aumentando también su percepción social mediante mecanismos puramente 
democráticos(Cabrera; Donoso.; Folgueiras y Luna, 2011, 100). Por lo tanto, a mayor participación, mayor educación para la ciudadanía.

Formación y preparación de la acción. En tercer lugar aparece la formación y la posible preparación de la acción. Es imprescindible tener en cuenta el hacer visible a los jóvenes participantes que el proyecto es suyo. Esto es posible si se explica el sentido del proyecto; es decir, que surge de una necesidad social y que con su actuación puede modificarse. De esta manera, la formación buscará una participación e implicación de los integrantes en las sesiones y a su vez, permitirá que formulen una opinión propia mediante la reflexión. Posteriormente a la adquisición de los conocimientos que se estiman necesarios, se les presenta el plan de acción para hacer posible un conjunto de actuaciones que puedan dar respuesta a la necesidad planteada. El plan de acción ha de estar firmado por el representante del grupo, tomando de esta forma la responsabilidad de trabajar en el proyecto.

Preparar y realizar el servicio. En cuarto lugar aparece el momento de preparar el servicio, es decir, una vez los jóvenes han asumido su participación han de pensar cómo verían posible dar respuesta a las demandas sociales planeadas y cuáles son los recursos de los que disponen. Han de pensar en las limitaciones y potencialidades de la realización del proyecto y su contexto. Después de haber diseñado los aspectos que quieren y pueden hacer, llega el momento de la acción. Así pues, se configura el servicio mediante los aprendizajes adquiridos y posteriormente se ponen en práctica. Es bueno, que a lo largo de este proceso de diseño y aplicación de las acciones pensadas por ellos, los educadores, profesores y maestros promuevan la reflexión y los jóvenes participantes vayan tomando conciencia del conjunto de aprendizajes que van surgiendo.

Reflexiones y agradecimientos. Al finalizar la acción llega el momento de aglutinar y recoger todo lo vivido, emociones, sentimientos, saberes, habilidades y aptitudes. Se busca poner orden a todo lo ocurrido. Es un espacio en el que los participantes van poniendo nombre al conjunto de sucesos que han ido apareciendo en su acción y de la cual, los aprendizajes cogen forma y fondo. Los anclan en una experiencia vivida que les permitirá, si ha sido significativa para ellos, retomar esos saberes cuando les sea necesario. Paralelamente, también aparecen los agradecimientos. Las actuaciones realizadas no han sido en vano, han sido significativas para los destinatarios de ellas, pero que a su vez, lo son para los implicados en el proyecto y, esto se celebra reconociendo y agradeciendo el trabajo realizado por ambas partes.

\section{Evaluar el desarrollo del proyecto y prever su continuidad}

Como en cualquier experiencia educativa, al finalizar el proceso aparece el momento de hacer balance. Es por tanto el momento de evaluar el programa, y esto será posible 


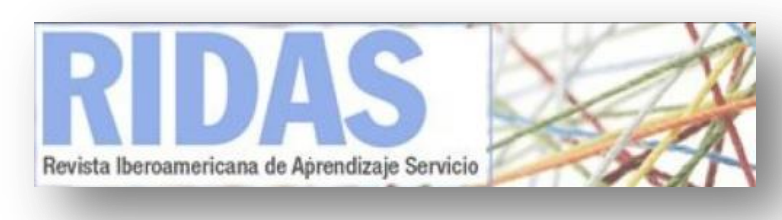

a partir de una evaluación transversal. Esta permitirá valorar si la experiencia ha sido positiva y si, en caso que se quiera, pueda volverse a hacer. Es por este motivo que también aparece la oportunidad de planificar posibles réplicas de éste. A continuación se especifica.

\section{Evaluar el desarrollo del proyecto y prever su continuidad}

Realizar una evaluación del proceso

Planificar próximas realizaciones del proyecto

Tabla 5. Evaluar el desarrollo del proyecto y prever su continuidad. Fuente: elaboración propia.

Realizar una evaluación del proceso. Cuando la aplicación del proyecto ya ha llegado a su fin, aparece este último, corto pero imprescindible periodo. Es el de la evaluación que ha de poderse hacer desde las múltiples vertientes empleadas. Se ha de valorar desde la perspectiva de: el referente de la entidad educativa, el referente de la entidad social, las entidades que puedan haber sido partícipes, los receptores del servicio, los ejecutores del servicio y el entorno (familiares, vecinos, compañeros).

Planificar próximas realizaciones del proyecto. En este momento es importante que los participante directos e indirectos valoren el proyecto mediante sus opiniones y aportando aspectos de mejora. De las personas que están más implicadas en este periodo, se podrían destacar los referentes. Entre ellos han de poder analizar el desarrollo del proyecto (los contactos, la formación, las acciones, y las reflexiones) y las posibilidades de replicarlo, si se considera oportuno, en la misma institución en próximos cursos.

De esta forma, en este periodo hay dos aspectos a trabajar: en primer lugar la opinión, valoración y análisis del proyecto, y en segundo lugar la probabilidad de la reproducción del mismo en la organización educativa, aunque dependa de muchos factores externos a ésta.

\section{Discusión y conclusiones}

Inicialmente, cuando se hablaba de aprendizaje servicio se vinculaba a un método que se trabaja principalmente en los centros educativos. Una de las primeras actividades que se hizo para introducir el aprendizaje servicio en nuestro país, fue detectar experiencias que ya se realizaban y que no se les denominaba de esta forma. Estas experiencias, a diferencia de muchas otras, tenían la peculiaridad de vincular el ámbito educativo con la sociedad. 


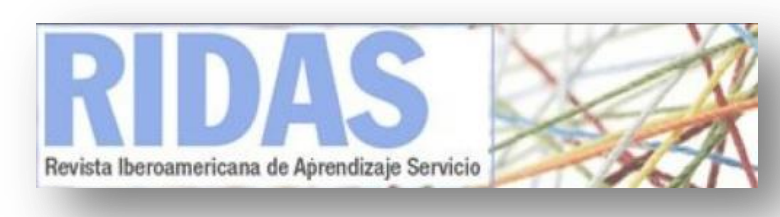

Al ver que la dimensión comunitaria aparece como un espacio relacional en el aprendizaje servicio, una de las hipótesis que surgieron se basaba en si era posible realizar experiencias de aprendizaje servicio impulsadas por entidades sociales. Tal como afirmaban Dewey (1957) o Freire (1987), todo lo que nos rodea educa, y si eso era cierto, las entidades sociales también pueden educar. Es por ello que a partir del aprendizaje servicio y de las necesidades sociales surge un proyecto con el Banco de Sangre y Tejidos que nos ha permitido visualizar una nueva forma de trabajar en la educación.

En este artículo se ha presentado un protocolo de actuación para desarrollar un proyecto de ApS desde de las entidades sociales. Un modo de llegar al mundo educativo desde las organizaciones sociales que favorezca a ambos ámbitos. El estudio y generalización del proyecto surgen de la demanda de crear un producto que paute una forma de actuar y de hacer. Se han detallado paso a paso cuáles son las fases y qué aspectos son indispensables para su aplicación.

Posiblemente es aventurada la afirmación que mediante la implantación de proyectos de aprendizaje servicio se transformen en educativas las organizaciones sociales. Pero sí que sería cierto sostener que una entidad como el Banco de Sangre y Tejidos está aplicando este proyecto formativo en múltiples centros escolares y de tiempo libre. No sólo proporciona saberes nuevos a las entidades educativas con las que trabaja, sino que facilita la apertura de estas hacia la comunidad. Así pues, no podemos negar que el Banco de Sangre y Tejidos tiene una forma de trabajar que puede ser replicable a otras entidades similares a ésta.

Aunque se haya presentado un protocolo de actuación general, solo se ha podido realizar durante estos últimos años desde el Banco de Sangre y Tejidos, es por ello que una de las prospectivas de dicha investigación sería poder realizarlo en otras entidades similares. Por lo tanto, poder aplicar este protocolo a situaciones parecidas, en las que se debería tener en cuenta que ambas entidades ganan, que el propósito de la organización social es paliar las necesidades sociales, y en los aspectos educativos que tiene la organización social para compartir con el mundo educativo.

Los proyectos de aprendizaje servicio son experiencias relacionadas con la comunidad que pueden ser impulsadas por entidades sociales o educativas y que requieren de unos pasos que son imprescindibles para su actuación. Estas fases se reúnen en un protocolo de actuación vinculado con las relaciones de partenariado que se crean entre las organizaciones que se vinculan, con el fin de mejorar la comunidad y aprender de forma controlada experiencias vividas. 


\section{Referencias Bibliográficas}

Bertrand,Y. \&Valois, P. (1995). Quinze pedagogs: la seva influència, avui. Barcelona: Universitat Oberta de Catalunya.

Cabrera, F.A., Donoso, T., Folgueiras, P. \& Luna, E. (2011). La participació ciutadana: dimensió clau de l'educació per a la ciutadania. Temps d'educació, 40, 97-116.

Dewey, J. (1957). Educación y democracia. Buenos Aires: Editorial Losada.

Freire, P. (1987). L'educació com a pràctica de la llibertat i altres escrits. Vic: Eumo.

Graell, M. (2010). Donació de sang i educació per a la ciutadania. Una experiencia de aprendizaje servicio. (Tesis Doctoral). Universitat de Barcelona, Barcelona, España.

Kemmis, S., \& McTaggart, R. (1988). Cómo planificar la investigación-acción. Barcelona: Laertes.

Larrauri, L, (2012). La educación segun John Dewey. Valencia: Tàndem Edicions.

Puig, J. M., Batlle, R., Bosch, C., \& Palos, J. (2006). Aprenentatge servei. educació per a la ciutadania. Barcelona: Octaedro.

Tapia, M. N. (2006). Aprendizaje y servicio solidario en el sistema educativo y las organizaciones juveniles. Buenos Aires: Ciudad Nueva.

Trilla, J., \& Novella, A. (2001). Educación y participación social de la infancia. Revista Iberoamericana De Educación, 26, 137-164.

Nota final

Este artículo forma parte del proyecto "Aprendizaje servicio y educación en valores", del Grupo de Investigación en Educación Moral de la Universidad de Barcelona, que ha sido financiado con una ayuda a la investigación concedida por el programa RecerCaixa 2010 (IP Josep M. Puig, no expediente 2010ACUP00219). 